\title{
On the Convergence Problem of One-Dimensional Hypersingular Integral Equations
}

\author{
Suzan J. Obaiys ${ }^{1,2}$ \\ ${ }^{1}$ Department of Mathematics, Faculty of Science, Universiti Putra Malaysia, 43400 Serdang, Selangor Darul Ehsan, Malaysia \\ ${ }^{2}$ Department of Mathematics, College of Education for Pure Sciences, Ibn AL-Haitham, Baghdad University, Baghdad, Iraq
}

Correspondence should be addressed to Suzan J. Obaiys; suzan_ye@yahoo.com

Received 8 July 2013; Accepted 18 September 2013

Academic Editor: Kue-Hong Chen

Copyright (c) 2013 Suzan J. Obaiys. This is an open access article distributed under the Creative Commons Attribution License, which permits unrestricted use, distribution, and reproduction in any medium, provided the original work is properly cited.

\begin{abstract}
We develop the expansion method of singular integral equation (SIE) for hypersingular integral equation (HSIE). Relating the hypersingular integrals to Cauchy principal-value integrals, we interpolate the kernel and the density functions to the truncated Chebyshev series of the second kind. The corresponding convergence results for the functions $f \in C^{\ell}([-1,1])$ and $K(t, x) \in$ $C^{\ell}([-1,1] \times[-1,1]), \ell \geq 1$, are derived in an appropriate $L_{2}[-1,1]$ norm to the true solution of the weight function. Numerical examples are also presented to validate the theoretical findings.
\end{abstract}

\section{Introduction}

The integral equation is defined as an equation with an unknown function that appears under the integral sign. These equations could be classified by the order of singularity [1]. Singular integral has been widely used and well developed [2-4]. A wealth of the literature on applications related to the numerical evaluation of hypersingular integral equations HSIEs could be found in [5-10]. This paper focuses on onedimensional singular integral equations (SIEs) found in various mixed boundary value problems of mathematical physics and engineering such as isotropic elastic bodies involving cracks, aerodynamics, hydrodynamics, elasticity, and other related areas.

Let us consider the hypersingular integral equation of the following form:

$$
\begin{aligned}
\frac{1}{\pi} f_{-1}^{1} \frac{Q(t)}{(t-x)^{2}} d t+\int_{-1}^{1} K(t, x) Q(t) d t & =f(x), \\
x & \in(-1,1),
\end{aligned}
$$

where $f(x)$ is a given function, the unknown function $Q$ satisfies the boundary conditions $Q( \pm 1)=0$, and the kernel function $K(t, x)$ satisfies a Hölder continuous first-derivative condition. The improper integral in the left side $f$ is defined as the finite part of the strongly singular integral in the sense of Hadamard which is defined as [11]

$$
f_{-1}^{1} \frac{f(t) d t}{(t-x)^{2}}=\lim _{\varepsilon \rightarrow 0}\left[\left(\int_{-1}^{x-\varepsilon}+\int_{x+\varepsilon}^{1}\right) \frac{f(t) d t}{(t-x)^{2}}-\frac{2 f(x)}{\varepsilon}\right] .
$$

One of the main concepts in the derivations is that the higher order singularity could be obtained from a lower order singularity by the accepted exchangeability of integration and differentiation [12]:

$$
f_{-1}^{1} \frac{f(t)}{(t-x)^{2}} d x=\frac{d}{d x} f_{-1}^{1} \frac{f(t)}{(t-x)} d t, \quad x \in(-1,1) .
$$

The objective of this paper is to develop an expansion method of HSIE in (1), for an effective error estimate, that is simple and easy to use. The numerical solution of HSIE of the first kind is given in Section 2. Section 3 presents the convergence of the numerical method in the class of function $H^{\alpha}([-1,1])$ and $L_{2}([-1,1])$. Moreover, the numerical results in Section 4 show that the constructed technique is fast and provides excellent accuracy where the error is almost zero. Section 5 presents the conclusion of this paper. 


\section{Description of the General Method}

Direct numerical treatment of (1) is not simple. Many researchers applied the classical method by approximating the unknown function $Q$ using the finite sum of Chebyshev polynomial of the second kind of the form:

$$
Q_{n}(t)=\sqrt{\left(1-t^{2}\right)} \sum_{i=0}^{n} C_{i} U_{i}(t), \quad|t|<1,
$$

where $C_{i}, i=0,1,2, \ldots, n$, are the unknown coefficients and $U_{n}(t)$ is the Chebyshev polynomial of the second kind:

$$
U_{n}(t)=\frac{\sin (n+1) \theta}{\sin \theta}, \quad t=\cos \theta, 0 \leq \theta \leq \pi .
$$

The second kind Chebyshev polynomial is orthogonal on $[-1,1]$ with the weight $w(t)=\sqrt{1-t^{2}}$ means

$$
\int_{-1}^{1} \sqrt{1-t^{2}} U_{i}(t) U_{j}(t) d t=\frac{\pi}{2} \delta_{i j},
$$

where $\delta_{i j}$ is the Kronecker delta and the recurrence relation is defined as follows:

$$
U_{n}(t)=2 t U_{n-1}(t)-U_{n-2}(t),
$$

with the initial values $U_{0}(t)=1$ and $U_{1}(t)=2 t$.

One could look for the hypersingular kernel in (1) in the form of the series approximation of the form [5]

$$
\frac{1}{(t-x)^{2}} \approx-2 \sum_{j=0}^{m}(j+1) U_{j}(x) U_{j}(t),
$$

while the regular kernel

$$
K(t, x)=\sum_{r=0}^{p} \rho_{r}(x) U_{r}(t),
$$

substituting (4), (8), and (9) into (1) and using the orthogonal property in (6) yield

$$
\sum_{i=0}^{n} C_{i}\left[-(i+1) U_{i}(x)+\frac{\pi}{2} \rho_{i}(x)\right]=f(x) .
$$

From the approximation (9) and by applying the scalar product to the functions $U_{j}(t)(j=0,1, \ldots, n)$ and integrating both sides with respect to $t$, we obtain the linear system

$$
\rho_{j}(x)=\frac{2}{\pi} \int_{-1}^{1} \sqrt{1-t^{2}} K(t, x) U_{j}(t) d t .
$$

Choosing the roots of $T_{n+1}(x)$ as the collocation points $x_{j}$ along the interval $[-1,1]$, which are

$$
x_{j}=\cos \left(\frac{2 j-1}{2(n+1)} \pi\right), \quad j=0,1, \ldots, n,
$$

one could reduce initial integral equation (1) to the finite linear algebraic system of $n+1$ linear equations with $n+1$ unknown coefficients $C_{i}$ of the form

$$
\sum_{i=0}^{n} C_{i}\left[-(i+1) U_{i}\left(x_{j}\right)+\frac{\pi}{2} \rho_{i}\left(x_{j}\right)\right]=f\left(x_{j}\right),
$$

where the calculation of $C_{i}$ endorses the evaluation of $Q_{n}(t)$ in (4).

\section{Convergence Rates}

In this section, we illustrate the steps of the error estimate for the approximate solution of our interest in HSIE of the form in (1). First, we recap the equation concerning the following operators:

$$
\begin{gathered}
H Q(x)=\frac{1}{\pi} f_{-1}^{1} \frac{\sqrt{1-t^{2}} Q(t)}{(t-x)^{2}} d t, \\
K Q(x)=\int_{-1}^{1} \sqrt{1-t^{2}} K(t, x) Q(t) d t .
\end{gathered}
$$

Taking into consideration (14), the operator equation of (1) could be rewritten as

$$
(H+K) Q(x)=f(x) .
$$

To solve a convergence problem of $Q_{n}(t)$, we should prove that $Q_{n}(t)$ satisfies the operator equation in (15).

Theorem 1. If $f(x) \in C^{\ell}[-1,1]$ and $k(t, x) \in C^{\ell}([-1,1] \times$ $[-1,1]), \ell \geq 1$, then

$$
\left\|Q-Q_{n}\right\|_{L_{2}, \sqrt{1-t^{2}}}=O\left(n^{-\ell}\right) .
$$

Proof. Using the relation $[12,13]$

$$
\int_{-1}^{1} \frac{\sqrt{\left(1-t^{2}\right)} U_{k}(t)}{(t-x)^{2}} d t=-\pi(k+1) U_{k}(t),
$$

along with the approximation in (4), yields

$$
H U_{k}=-(k+1) U_{k} \text {. }
$$

Let us denote the space $L_{2, w}$ of real valued functions square integrable with respect to the weight function $w=\sqrt{1-x^{2}}$ as

$$
L_{2, w}=\left\{\left.h(x)\left|\int_{-1}^{1} w(x)\right| h(x)\right|^{2} d x<\infty\right\} .
$$

Based on (19), the inner product might be defined as follows:

$$
\langle Q, g\rangle_{w}=\int_{-1}^{1} Q(x) g(x) w(x) d x, \quad \forall Q, g \in L_{2, w}
$$

The set $\left\{U_{k}\right\}_{k=0}^{\infty}$ is the orthogonal basis in $L_{2, w}$, so that if $u \in$ $L_{2, w}$ then

$$
u=\sum_{n=0}^{\infty}\left\langle u, U_{n}\right\rangle_{w} U_{n}
$$

where the sum converge in $L_{2, w}$. Then we would need to use a subspace of $L_{2, w}$ which contains all $u$, such that

$$
\sum_{n=0}^{\infty}(n+1)^{2}\left\langle u, U_{n}\right\rangle_{w}^{2}<\infty
$$


We define this set by $L_{1, w}$, and it is made into Hilbert space by the following inner product:

$$
\langle u, v\rangle_{1}=\sum_{n=0}^{\infty}(n+1)^{2}\left\langle u, U_{n}\right\rangle_{w}\left\langle v, U_{n}\right\rangle_{w}
$$

where the norm of $v \in L_{1, w}$ is defined by

$$
\|u\|_{1}^{2}=\sum_{n=0}^{\infty}(n+1)^{2}\left\langle u, U_{n}\right\rangle_{w}^{2} .
$$

Assume that $v_{n}=\left(U_{n} /(n+1)\right)$ and note that $\left\|v_{n}\right\|_{1}=1$, therefore $\left\{v_{n}\right\}_{n}^{\infty}=0$ from an orthonormal basis of $L_{1, w}$, and if $u \in L_{1, w}$ then

$$
u=\sum_{n=0}^{\infty}\left\langle u, v_{n}\right\rangle_{1} v_{n}
$$

To show that $H^{-1}: L_{2, w} \rightarrow L_{1, w}$ is unitary, let us consider expansions of $f \in L_{2, w}$ and $g \in L_{1, w}$. It is not difficult to see that

$$
H f(x)=\sum_{n=0}^{\infty}-(n+1) C_{n} U_{n}(x)=g(x),
$$

where $C_{n}=\left\langle f, U_{n}\right\rangle$, and we use the expansion of $g$, such that

$$
g(x)=\sum_{n=0}^{\infty} b_{n} U_{n}(x) .
$$

Then $-(n+1) C_{n}=b_{n}$ which gives

$$
C_{n}=-\frac{b_{n}}{n+1} \text {. }
$$

So,

$$
f(x)=\sum_{n=0}^{\infty}-\frac{b_{n}}{n+1} U_{n}(x) .
$$

We have $f=H^{-1} g$, and by using (29), becomes

$$
f=H^{-1} g(x)=\sum_{n=0}^{\infty}-\frac{b_{n}}{n+1} U_{n}(x) .
$$

For the approximation in (4), the coefficients $\left\{C_{i}\right\}_{i=0}^{n}$ satisfy

$$
\left\langle H Q_{n}+K Q_{n}-f, Q_{k}(x)\right\rangle=0, \quad k=0,1,2, \ldots
$$

To show that $\left\{Q_{n}\right\}$ converges, it should verify the operator equation in (15).

Let $P_{n}$ be the projection operator to the span of $\left\{U_{0}, U_{1}, \ldots, U_{n}\right\}$; then, the orthogonal projection space $P_{n}$ : $L_{2, w} \rightarrow L_{2, w}$, satisfies [14]

$$
P_{n}\left(H Q_{n}+K Q_{n}-f\right)=0 .
$$

Since $P_{n} H Q_{n}=H Q_{n}$, (32) becomes

$$
\begin{gathered}
H Q_{n}+P_{n} K Q_{n}=P_{n} f, \\
H Q+P_{n} K Q=f-K Q+P_{n} K Q, \\
\left(H+P_{n} K\right) Q=f-K Q+P_{n} K Q .
\end{gathered}
$$

We know that

$$
\left(H+P_{n} K\right) Q_{n}=P_{n} f
$$

which gives

$$
\begin{aligned}
\left(H+P_{n} K\right)\left(Q-Q_{n}\right) & =f-K Q+P_{n} K Q-P_{n} f \\
& =H Q-P_{n} H Q .
\end{aligned}
$$

$P_{n}: L_{2, w} \rightarrow \Omega=\operatorname{span}\left\{\phi_{1}, \phi_{2}, \ldots, \phi_{n}\right\}$. Let $g \in L_{2, w}$, then $P_{n} g=h=C_{1} \phi_{1}+C_{2} \phi_{2}+\cdots+C_{n} \phi_{n}$. From (18), we could extend $H$ as a bounded operator from $L_{2}(p)$ to $L_{1, w}$; then using (20),

$$
P_{n}\left((H+K) Q_{n}-f\right)=0
$$

Since

$$
P_{n}\left((H+K) Q_{n}-f\right)=\sum_{k=0}^{n} \alpha_{k} \phi_{k}, \quad \alpha_{k} \neq 0
$$

then

$$
\left\langle(H+K) Q_{n}-f, \phi_{k}\right\rangle=0, \quad k=0,1,2, \ldots, n .
$$

Applying the approximate solution in (4) to the inner product in (20), so that

$$
\left\langle H Q_{n}+K Q_{n}-f, U_{k}\right\rangle=0,
$$

then the bounded operator $H$ could be written as

$$
\begin{gathered}
\sum_{k=0}^{n}-(k+1) C_{k} U_{k}(x)+\sum_{k=0}^{n} g_{k}(t) U_{k}(x)=\left\langle f, U_{k}\right\rangle, \\
\left\langle f, \phi_{k}\right\rangle=\sum_{k=0}^{n}\left(g_{k}(t)-(k+1) C_{k}\right) U_{k}(x), \\
\left\langle h, U_{k}\right\rangle=0 .
\end{gathered}
$$

We define the projection $\Omega_{n}$ such that

$$
\begin{gathered}
\Omega_{n}=\left\{h \mid h \perp U_{k}, k=0,1, \ldots, n\right\}, \\
L_{2, w}=\Omega_{1} \oplus \Omega_{2} \longrightarrow h=h_{1}+h_{2}, \\
\Omega_{1}=\left\{h_{1} \in L_{2, w} \mid h_{1}=\sum_{k=0}^{n} C_{k} \phi_{k}\right\}=\operatorname{span}\left\{Q_{1}, \ldots, Q_{n}\right\}, \\
\Omega_{2}=\left\{h_{2} \mid h_{2} \perp h_{1} ; h_{1} \in \Omega_{1}\right\}, \\
P_{n}: L_{2, w} \longrightarrow \Omega_{1},
\end{gathered}
$$

then

$$
H Q_{n}=\sum_{k=0}^{n}-(k+1) C_{k} U_{k}(x) \in \Omega_{1} .
$$

Since $H Q \in \Omega_{1}, P_{n}$ is a linear operator that gives (35). 
For $H^{-1}$,

$$
\begin{aligned}
H g & =-\sum_{k=0}^{n}(k+1) g_{k} U_{k}(x) \\
& \Longrightarrow H^{-1} h=\sum_{k=0}^{n} \frac{1}{-(k+1)} h_{k} U_{k}(x) .
\end{aligned}
$$

It is known that $H * H^{-1}=I$.

Let $\phi \in L_{2, w}$ and $\phi=h_{1}+h_{2}$; then the projection operator $P_{n}$ is as follows:

$$
P_{n} \phi=h_{1} \subset \Omega_{1} .
$$

It is well known that $P_{n}$ is bounded operator:

$$
\left\|P_{n} \phi\right\| \leq\|\phi\|, \quad \forall \phi \in L_{2, w} .
$$

Let us consider $H+P_{n} K$ operator in $L_{2, w}$ :

$$
\left\|P_{n} K\right\|<1 \text {. }
$$

We choose $n_{0}$, such that for all $n \geq n_{0}$, one has

$$
\left\|P_{n} \phi\right\|<1 \text {. }
$$

Let us fix the element, $\phi \in L_{2, w}$, and by considering the operator:

$$
B g=H^{-1}\left(\phi-P_{n} K g\right) .
$$

This operator is contraction:

$$
\left\|B g_{1}-B g_{2}\right\|<\alpha\left\|g_{1}-g_{2}\right\|, \quad 0<\alpha<1 .
$$

Since

$$
\begin{gathered}
\left\|H^{-1} \phi-H^{-1} P_{n} K g_{1}-H^{-1} \phi+H^{-1} P_{n} K g_{2}\right\| \\
=\left\|H^{-1} P_{n} K\left(g_{2}-g_{1}\right)\right\| \\
\leq\left\|H^{-1} P_{n} K\right\|\left\|g_{2}-g_{1}\right\|, \\
\alpha=\left\|H^{-1} P_{n} K\right\|<1 .
\end{gathered}
$$

Since $B$-contraction operator, then it has an unique fixed point $g$ :

$$
g=H^{-1}\left(\phi-P_{n} K g\right) .
$$

By applying $H$ to both sides, we obtain

$$
H g=\phi-P_{n} K g
$$

or

$$
\phi=H g+P_{n} K g=\left(H+P_{n} K\right) g .
$$

For any $\phi \in L_{2, w}$, there exists an unique $g \in L_{2, w}$, such that $\phi=\left(H+P_{n} K\right) g$, which means that $\left(H+P_{n} K\right)^{-1}$ exists:

$$
\begin{gathered}
\left(H+P_{n} K\right)\left(Q-Q_{n}\right)=H Q-P_{n} H Q, \\
Q-Q_{n}=\left(H+P_{n} K\right)^{-1}\left(H Q-P_{n} H Q\right) .
\end{gathered}
$$

To show that our operator $H+P_{n} K$ is bounded, we apply the following theorem [15].
Theorem 2. Let L be a Banach space, and let I be the identity operator on L. Suppose that $A$ is a bounded linear operator mapping L into itself, such that

$$
\|A\|<1 .
$$

Then the operator $(I-A)^{-1}$ that exists is bounded and could be represented in the form

$$
(I-A)^{-1}=\sum_{k=0}^{\infty} A^{k} .
$$

For the details of Theorem 2, see [15].

Let us consider that

$$
H+P_{n} K=H\left(I+H^{-1} P_{n} K\right) .
$$

Obviously we have

$$
\left\|H^{-1} P_{n} K\right\|<1
$$

It follows that

$$
\begin{aligned}
\left(H+P_{n} K\right)^{-1} & =\left(H\left(I+H^{-1} P_{n} K\right)\right)^{-1} \\
& =\left(I+H^{-1} P_{n} K\right)^{-1} H^{-1} \\
& =\left(\sum_{j=0}^{\infty}(-1)^{j}\left(H^{-1} P_{n} K\right)^{j}\right) H^{-1} .
\end{aligned}
$$

Applying Theorem 2 yields

$$
\left(H+P_{n} K\right)^{-1}=\sum_{j=0}^{\infty} H^{-j-1}\left(P_{n} K\right)^{j} .
$$

The norm would be

$$
\begin{aligned}
\left\|\left(H+P_{n} K\right)^{-1}\right\| & \leq \sum_{j=0}^{\infty}\left\|H^{-j-1}\left(P_{n} K\right)^{j} \phi\right\| \\
& \leq \sum_{j=0}^{\infty}\left\|H^{-1}\right\| \cdot\left\|H^{-1} P_{n} K\right\|^{j} .
\end{aligned}
$$

Since

$$
\left\|H^{-1} P_{n} K\right\|=q<1,
$$

then

$$
\begin{gathered}
\left\|\left(H+P_{n} K\right)^{-1}\right\|=\left\|H^{-1}\right\| \sum_{j=0}^{\infty} q^{j}=\left\|H^{-1}\right\| \frac{1}{1-q}, \\
\left\|\left(H+P_{n} K\right)^{-1}\right\|=\frac{\left\|H^{-1}\right\|}{1-\left\|H^{-1} P_{n} K\right\|} \\
=C \\
\left\|Q-Q_{n}\right\|_{2, w} \leq\left\|\left(H+P_{n} K\right)^{-1}\right\| \cdot\left\|H Q-P_{n} H Q\right\|_{2, w} \\
\leq C\left\|H Q-P_{n} H Q\right\|_{2, w} .
\end{gathered}
$$


Applying (19) to (60) gives

$$
\begin{aligned}
\left\|Q-Q_{n}\right\|_{2} & =C\left(\int_{-1}^{1}\left|H Q-P_{n} H Q\right|^{2} w(x) d x\right)^{1 / 2} \\
& \leq C\left(\int_{-1}^{1}\left|H Q(x)-\eta_{n}(x)\right|^{2} w(x) d x\right)^{1 / 2} \\
& \leq C\left\|H Q(x)-\eta_{n}(x)\right\|_{L_{\infty}}\left(\int_{-1}^{1} w(x) d x\right)^{1 / 2} .
\end{aligned}
$$

Applying Jackson's theorem [14] yields

$$
\left\|Q-Q_{n}\right\|_{2}=C\left(n^{-\ell}\right) \text {. }
$$

From (64), Theorem 1 is proved.

\section{Numerical Example}

Here, we illustrate the above method to obtain approximate numerical solution of Fredholm integral equation.

Example 1. We consider Fredholm full equation of the form

$$
\begin{aligned}
\frac{1}{\pi} f_{-1}^{1} & \frac{\sqrt{1-t^{2}} g(t)}{(t-x)^{2}} d t \\
& +\frac{16}{\pi} \int_{-1}^{1} \sqrt{1-t^{2}} x^{3} t^{3} g(t) d t=16 x-31 x^{3} .
\end{aligned}
$$

The exact solution of (66) is

$$
g(t)=8 t^{3}-4 t
$$

By using the method in Section 2, if we approximate $g(t)$ using Chebyshev polynomials of the second kind,

$$
g(t)=\sum_{i=0}^{n} C_{i} U_{i}(t)
$$

and $C_{i}$ satisfies the linear system in (10).

According to (11) and (7),

$$
\rho_{j}(x)=\frac{4 x^{3}}{\pi^{2}} \int_{-1}^{1} \sqrt{1-t^{2}}\left[U_{3}(t)+2 U_{1}(t)\right] U_{j}(t) d t .
$$

Using the orthogonal property in (6), we obtain

$$
\begin{gathered}
\rho_{0}(x)=0, \quad \rho_{1}(x)=\frac{4 x^{3}}{\pi}, \\
\rho_{2}(x)=0, \quad \rho_{3}(x)=\frac{2 x^{3}}{\pi}, \\
\rho_{j}(x)=0, \quad j \geq 4 .
\end{gathered}
$$

Substituting the above result in the system of equations into (13) for $n=3$ gives

$$
\begin{aligned}
& \sum_{i=0}^{3} C_{i}\left[-(i+1) U_{i}\left(x_{j}\right)\right]+2 C_{1} x_{j}^{3}+C_{3} x_{j}^{3} \\
& \quad=16 x_{j}-31 x_{j}^{3}, \quad i=0,1,2,3 \\
& \left(-31 C_{3}+2 C_{1}\right) x_{j}^{3}-12 C_{2} x_{j}^{2}+\left(16 C_{3}-4 C_{1}\right) x_{j} \\
& \quad+3 C_{2}-C_{0}=16 x_{j}-31 x_{j}^{3}, \quad j=0,1,2,3 .
\end{aligned}
$$

Using the comparison method for solving the above system of equation leads to

$$
\begin{aligned}
& -31 C_{3}+2 C_{1}=-31, \quad-12 C_{2}=0, \\
& 16 C_{3}-4 C_{1}=16, \quad 3 C_{2}-C_{0}=0,
\end{aligned}
$$

then the solution of the above system is

$$
C_{0}=C_{1}=C_{2}=0, \quad C_{3}=1 .
$$

Substituting the values of (73) into (68) gives the numerical solution of (66) which is identical to the exact solution.

Example 2. We solve Fredholm HSIE of the form

$$
\begin{gathered}
\frac{1}{\pi} \int_{-1}^{1} \frac{Q(t)}{(t-x)^{2}} d t+\int_{-1}^{1} \frac{e^{x}}{t-x} Q(t) d t \\
=\pi e^{x}\left(x-4 x^{3}\right)-12 x^{2}+1 .
\end{gathered}
$$

Note that, by the help of (3), the exact solution of (74) is

$$
Q(x)=\sqrt{1-x^{2}}\left(4 x^{2}+1\right) \text {. }
$$

From (11),

$$
\rho_{i}(x)=\frac{2}{\pi} \int_{-1}^{1} \sqrt{1-t^{2}} \frac{e^{x}}{t-x} U_{i}(t) d t .
$$

It is well known that $[12,13]$

$$
\int_{-1}^{1} \frac{\sqrt{1-t^{2}} U_{i-1}(t)}{t-x} d x=-\pi T_{i}(x)
$$

so that

$$
\rho_{i}(x)=-2 e^{x} T_{i+1}(x),
$$

where $T_{i}$ is the Chebyshev polynomial of the first kind defined:

$$
T_{n}(t)=\cos n \theta, \quad t=\cos \theta, 0 \leq \theta \leq \pi .
$$

This polynomial is orthogonal on $[-1,1]$ with respect to $w(t)=\left(1 / \sqrt{1-t^{2}}\right)$, and the terms could be found using the recurrence relation:

$$
T_{n}(t)=2 t T_{n-1}(t)-T_{n-2}(t),
$$


TABLE 1: The error of the numerical solution of (74).

\begin{tabular}{lccc}
\hline & \multicolumn{2}{c}{$N=4$} & \\
$x$ & Error & $x$ & Error \\
\hline-1 & 0 & 1 & 0 \\
-0.998 & $4.7 e^{-013}$ & 0.998 & $4.7 e^{-013}$ \\
-0.8 & $3.4 e^{-011}$ & 0.8 & $3.1 e^{-011}$ \\
-0.7 & $2.1 e^{-011}$ & 0.7 & $2.1 e^{-011}$ \\
-0.6 & $2.3 e^{-011}$ & 0.6 & $2.2 e^{-011}$ \\
-0.5 & $2.6 e^{-011}$ & 0.5 & $2.6 e^{-011}$ \\
-0.4 & $2.7 e^{-011}$ & 0.4 & $2.2 e^{-011}$ \\
-0.3 & $3.1 e^{-011}$ & 0.3 & $3.2 e^{-011}$ \\
-0.2 & $2.2 e^{-011}$ & 0.2 & $2.2 e^{-011}$ \\
-0.1 & $3.3 e^{-011}$ & 0.1 & $3.3 e^{-011}$ \\
0.0 & $2.8 e^{-011}$ & 0.0 & $2.8 e^{-011}$ \\
\hline
\end{tabular}

where $T_{0}(t)=1$ and $T_{1}(t)=t$ are the starting values of (80), which provides the values of $\rho_{i}(x)$ for a certain $i$ :

$$
\begin{gathered}
\rho_{0}(x)=-2 x e^{x}, \quad \rho_{1}(x)=-2\left(2 x^{2}-1\right) e^{x}, \\
\rho_{2}(x)=-2\left(4 x^{3}-3 x\right) e^{x}, \\
\rho_{3}(x)=-2\left(8 x^{4}-8 x^{2}+1\right) e^{x}, \ldots
\end{gathered}
$$

Substituting the values of (81) into the system of (13) for $n=4$ gives

$$
\begin{aligned}
\sum_{i=0}^{4} C_{i} & {\left[-(i+1) U_{i}\left(x_{j}\right)+\frac{\pi}{2} \rho_{i}\left(x_{j}\right)\right] } \\
= & \pi e^{x_{j}}\left(x_{j}-4 x_{j}^{3}\right)-12 x_{j}^{2}+1, \quad j=0,1,2,3,4 .
\end{aligned}
$$

Using (12) as a collocation points for $j=4$, give the required system of unknown coefficients $C_{i}, i=0, \ldots, 4$. The solution of the system in (82) gives

$$
C_{0}=2, \quad C_{2}=1, \quad C_{1}=C_{3}=C_{4}=0 .
$$

Substituting the above values into (4), we obtain the numerical solution of (74), which is identical to the exact solution. The error of the numerical solution (4) of (74) is given in Table 1.

\section{Conclusion}

We have investigated the numerical solutions for the bounded hypersingular integral equation of the first kind using an efficient expansion method. We are able to prove the convergence of our method for the function $f \in C^{\ell}([-1,1])$ in the class of functions $H^{\alpha}([-1,1])$ and $L_{2}([-1,1])$. Moreover, the error in (65) decreases very quickly and the convergence is very fast to the exact solution even when $x$ is close to the end points. In particular, if $\ell$ in the relation (65) can be chosen to be any large positive number, then the error decreases rapidly as $n$ increases. Then the sequence $\left\{Q_{n}\right\}$ converges uniformly in $L_{2, w}$ norm to $\{Q\}$. The numerical examples give the exact solution for different kernels $K(x, t)$ and the unknown function $Q$. Therefore, referring to the computed numerical solution, we are able to obtain a very good convergence for any singular point $x \in(-1,1)$. MATLAB codes are developed to obtain the numerical results where the numerical experiments reaffirm the theoretical results.

\section{References}

[1] M. Rahman, Integral Equations and Their Applications, WIT Press, Great Britain, UK, 2007.

[2] J. Helsing, "A fast and stable solver for singular integral equations on piecewise smooth curves," SIAM Journal on Scientific Computing, vol. 33, no. 1, pp. 153-174, 2011.

[3] T. Hasegawa and T. Torii, "An automatic quadrature for Cauchy principal value integrals," Mathematics of Computation, vol. 56, no. 194, pp. 741-754, 1991.

[4] P. A. Martin, F. J. Rizzo, and T. A. Cruse, "Smoothnessrelaxation strategies for singular and hypersingular integral equations," International Journal for Numerical Methods in Engineering, vol. 42, no. 5, pp. 885-906, 1998.

[5] S. J. Obaiys, Z. K. Eskhuvatov, N. M. A. Nik long, and M. A. Jamaludin, "Galerkin method for the numerical solution of hypersingular integral equations based Chebyshev polynomials," International Journal of Mathematical Analysis, vol. 6, no. 53-56, pp. 2653-2664, 2012.

[6] H. K. Hong and J. T. Chen, "Derivations of integral equations of elasticity," Journal of Engineering Mechanics, vol. 114, no. 6, pp. 1028-1044, 1988.

[7] J. T. Chen and H. K. Hong, "Review of dual boundary element methods with emphasis on hypersingular integrals and divergent series," Applied Mechanics Reviews, vol. 52, no. 1, pp. 17-32, 1999.

[8] H. Ammari, G. Bao, and A. W. Wood, "An integral equation method for the electromagnetic scattering from cavities," Mathematical Methods in the Applied Sciences, vol. 23, no. 12, pp. 1057-1072, 2000.

[9] G. Krishnasamy, L. W. Schmerr, T. J. Rudolphi, and F. J. Rizzo, "Hypersingular boundary integral equations: some applications in acoustic and elastic wave scattering," Journal of Applied Mechanics, vol. 57, no. 2, pp. 404-414, 1990.

[10] Y. Z. Chen, "A numerical solution technique of hypersingular integral equation for curved cracks," Communications in Numerical Methods in Engineering, vol. 19, no. 8, pp. 645-655, 2003.

[11] I. K. Lifanov, L. N. Poltavskii, and G. M. Vainikko, Hypersingular Integral Equations and Their Applications, vol. 4, CRC Press LLC, 2004.

[12] S. J. Obaiys, Z. K. Eshkuvatov, and N. M. A. Nik Long, "On error estimation of automatic quadrature scheme for the evaluation of Hadamard integral of second order singularity," UPB Scientific Bulletin, Series A, vol. 75, no. 1, pp. 85-98, 2013.

[13] J. C. Mason and D. C. Handscomb, Chebyshev Polynomials, CRC Press LLC, 2003.

[14] M. A. Golberg, "The convergence of several algorithms for solving integral equations with finite part integrals. II," Applied Mathematics and Computation, vol. 21, no. 4, pp. 283-293, 1987.

[15] A. N. Kolmogorov and S. V. Fomīn, Introductory Real Analysis, Dover Publications, New York, NY, USA, 1975. 


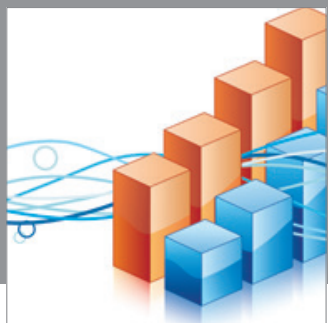

Advances in

Operations Research

mansans

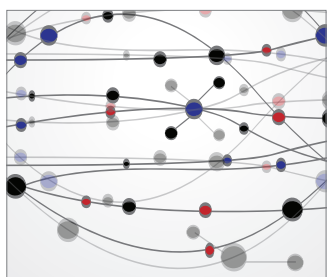

The Scientific World Journal
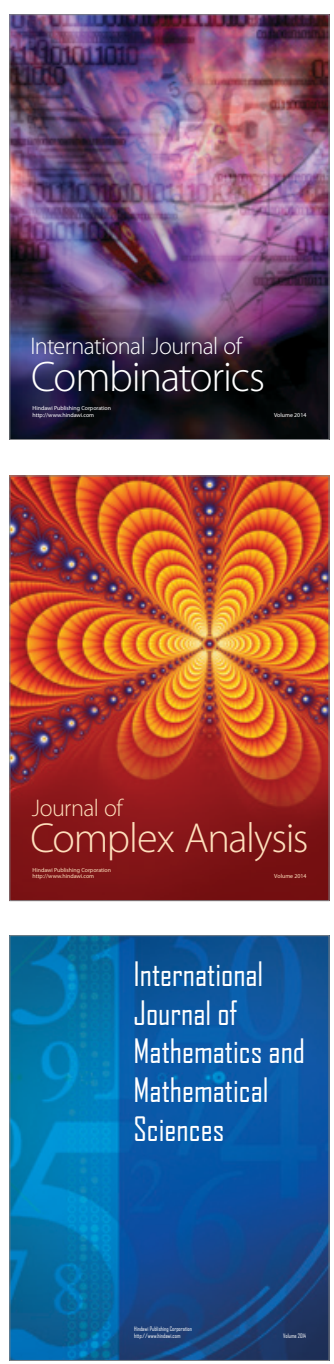
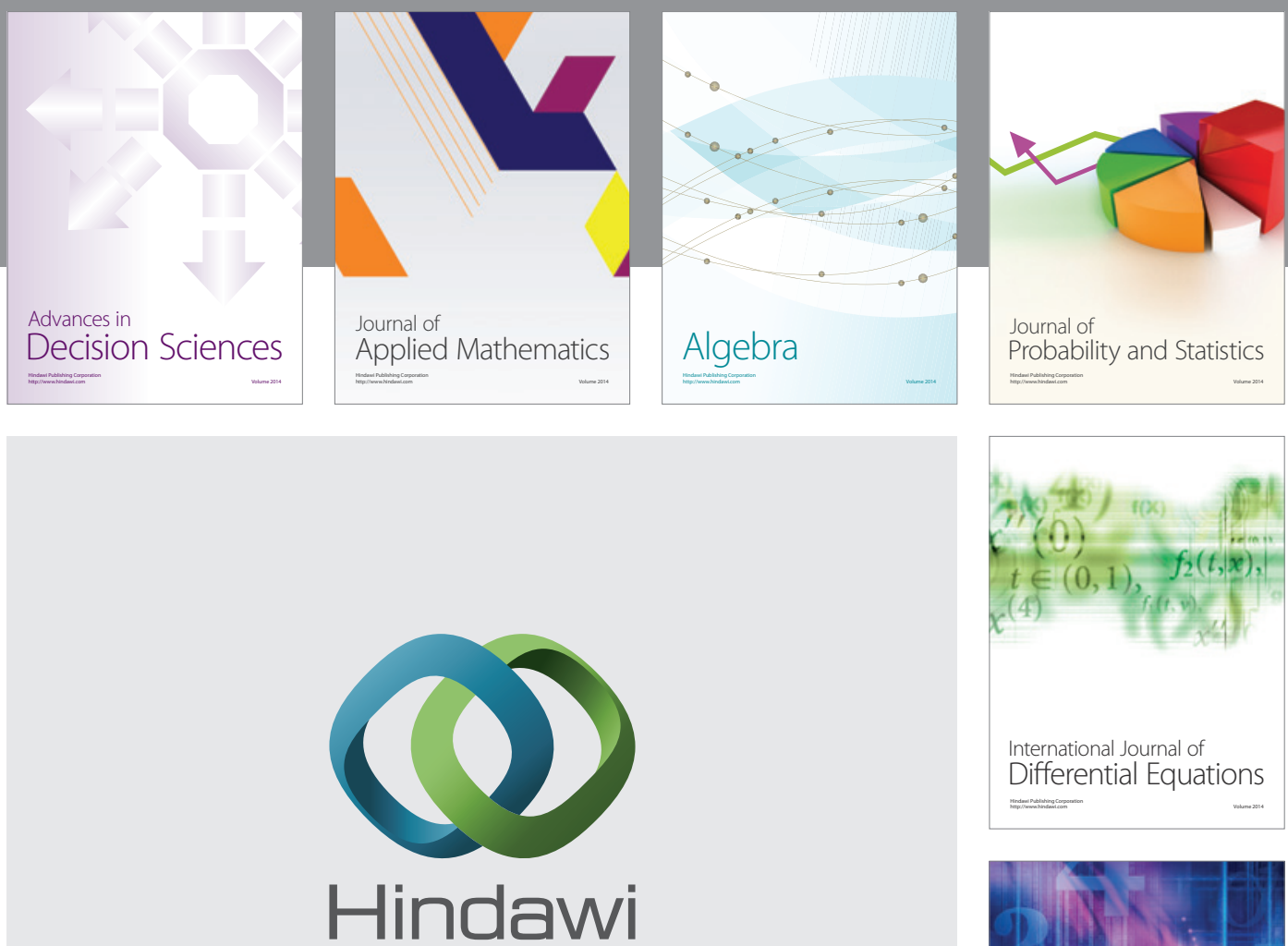

Submit your manuscripts at http://www.hindawi.com
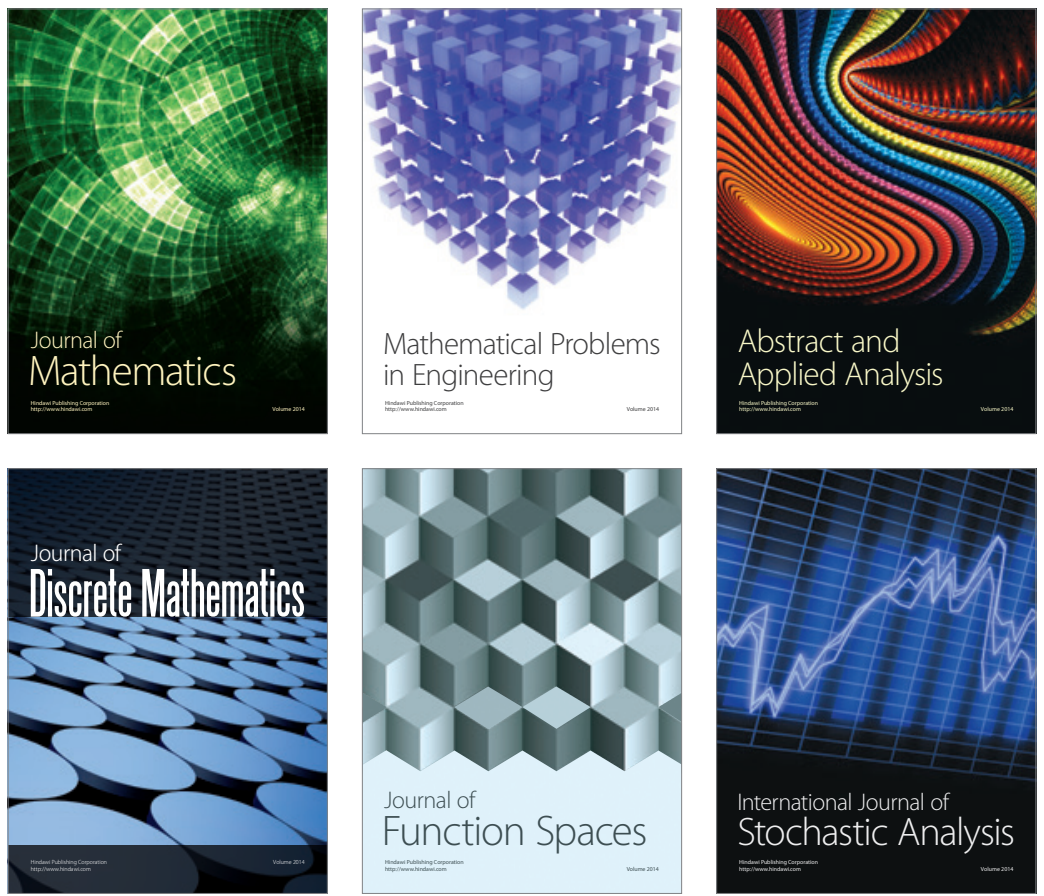

Journal of

Function Spaces

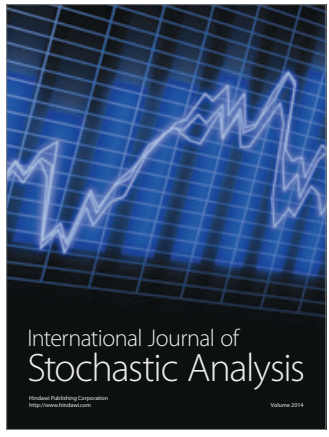

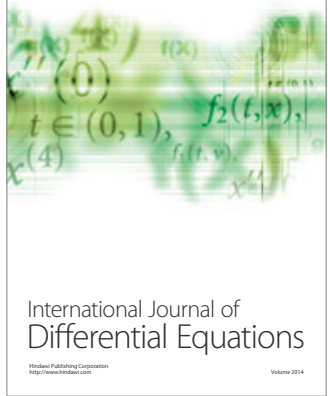
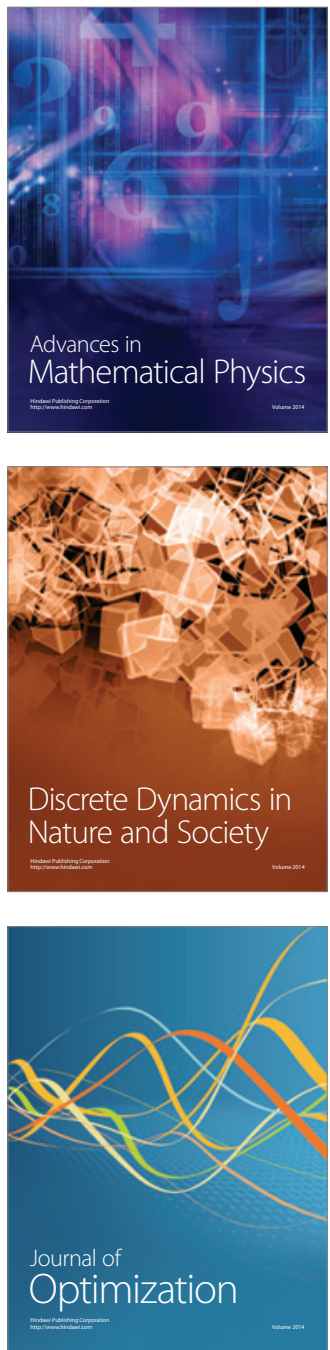